\title{
Digit ratio (2D:4D) and behavioral differences between inbred mouse strains
}

\author{
A. A. Bailey ${ }^{\dagger}$, D. Wahlsten ${ }^{\ddagger}$ and P. L. Hurd ${ }^{*}, \dagger$ \\ ${ }^{\dagger}$ Department of Psychology, University of Alberta, Edmonton, \\ Alberta, and \\ ${ }^{\ddagger}$ Great Lakes Institute, University of Windsor, Windsor, Ontario, \\ Canada \\ "Corresponding author: P. L. Hurd, Department of Psychology, \\ Biological Sciences Building P217, University of Alberta, Edmonton, \\ Alberta, T6G 2E9 Canada. E-mail: phurd@ualberta.ca
}

Digit ratio (2D:4D) is a trait, which is sexually differentiated in a variety of species. In humans, males typically have shorter second digits (2Ds) (index fingers) compared to fourth digits (4Ds) (ring fingers) whereas females' fingers are more equal in length. Smaller, more masculine, digit ratios are thought to be associated with higher prenatal testosterone levels, greater sensitivity to prenatal androgens or both. Men with more masculine digit ratios have shown increased ability, achievement and speed in sports and tend to report that they are more physically aggressive. Previous research has shown the same sexually differentiated pattern in the hind paws of laboratory mice as in human hands, males have lower 2D:4D than females. We measured hind paw digit ratio in mice of eight inbred strains. These measurements were made while blind to strain, sex and whether the paw was from the left or right side. We found large differences in digit ratio between the strains and suggest that inbred mice are a promising system for investigating the correlation between digit ratio and behavioral traits.

Keywords: 2D:4D, digit ratio, inbred mouse strains, prenatal testosterone

Received 16 August 2004, revised 29 September 2004, accepted for publication 15 October 2004

Many species show sexual differentiation in the ratio of second digit (2D) (index finger) to fourth digit (4D) (ring finger). In humans, males typically have smaller finger length ratios (i.e. shorter index fingers than ring fingers) than females (Manning 2002a; Manning et al. 2000; Peters et al. 2002). Hand bone proportions are fixed in utero, and digit ratio (2D:4D) is thought to remain stable during development (Brown et al. 2002a; Garn et al. 1975; Manning 2002a; Manning et al. 1998).
Variation in digit ratio appears to be influenced by prenatal testosterone during development (Manning 2002a; Manning et al. 2003a). Humans (both male and female) with elevated fetal androgens due to congenital adrenal hyperplasia exhibit lower, more masculine, finger length ratios than controls (Brown et al. 2002b; Okten et al. 2002). Two causal mechanisms have been conjectured to explain this phenomenon. The first is that common genes (Hoxa and Hoxd) underlie the development of both digits and gonads (Kondo et al. 1997; Peichel et al. 1997) such that the timing of gene regulation leaves a digit ratio that reflects the quantity of androgen produced. The second proposed mechanism is that finger ratio is a function of androgen sensitivity rather than androgen concentration. Androgen receptor alleles with fewer terminal domain CAG repeats produce receptors with higher androgen sensitivity (Chamberlain et al. 1994; Kazemi-Esfarjani et al. 1995) and are associated with lower finger ratios (Manning et al. 2003a).

Hind paws in laboratory mice have also been found to follow the same sexually differentiated pattern as human hands (Brown et al. 2002a; Manning et al. 2003b). Inbred mouse strains are valuable models for investigating the causes of individual variation in behavior (Flint 2003; Wahlsten et al. 2003). Considerable variation exists between strains in behavior. Because each strain is genetically uniform, variation within strains is due to environmental or gene-environment interaction effects. The purpose of the present study was to test for variation in digit ratio across a number of inbred strains to assess the potential of inbred mouse strains for exploring organizational effects of sex hormones on behavioral traits.

\section{Materials and methods}

Subjects were 184 male and female adult mice (approximately 14-16 weeks old), from eight different inbred strains. These mice were all euthanized at the conclusion of an unrelated study in accordance with all applicable laws and guidelines and as approved by the University of Alberta's Biological Sciences Animal Services' ethics review committee. All individuals were purchased from the same supplier (The Jackson Laboratory, Bar Harbor, ME) and were all subjected to the same housing conditions and tests prior to euthanasia. The strains and the sample sizes per sex were 129S1/SvImJ (12 males, 11 females), A/J (12 males, 11 females), BTBR_+T_tf/tf (12 males, 11 females), BALB/cByJ (11 males, 12 females), C3H/HeJ (12 males, 11 females), 
C57BL/6J (12 males, 12 females), DBA/2J (11 males, 12 females) and FVB/NJ (11 males, 12 females). All paws were removed after death and preserved in a $10 \%$ formalin solution.

Paws were placed palm up and stuck to a piece of adhesive backing to ensure digits were completely flat. We chose this method because it was more effective than pressing the paws with a glass slide, which created a reflection, obscured paw details and did not completely flatten the digits. Paws were photographed under a Wild M3Z stereoscope (Leica) with a Cohu 4815 video camera (Cohu, San Diego, CA) using an 8-bit gray scale. The software package (GLOBAL LABORATORY IMAGING) was used to capture, sharpen and save images for later analysis. The first sharpening filter was applied to all images. The GNU IMAGE MANIPULATION PROGRAM measure tool was used to measure all images. All left paw images were digitally translated along the vertical axis to appear as right paws. Images were assigned random identifiers so as to render the scorers blind to individual, strain or paw side. The length, in pixels, of the 2D and 4D for each paw was measured from the mid-point of the basal crease to the tip of the digit, excluding the nail (Fig. 1). Digit ratios were calculated by dividing the length of the $2 \mathrm{D}$ by the length of the $4 \mathrm{D}$. The

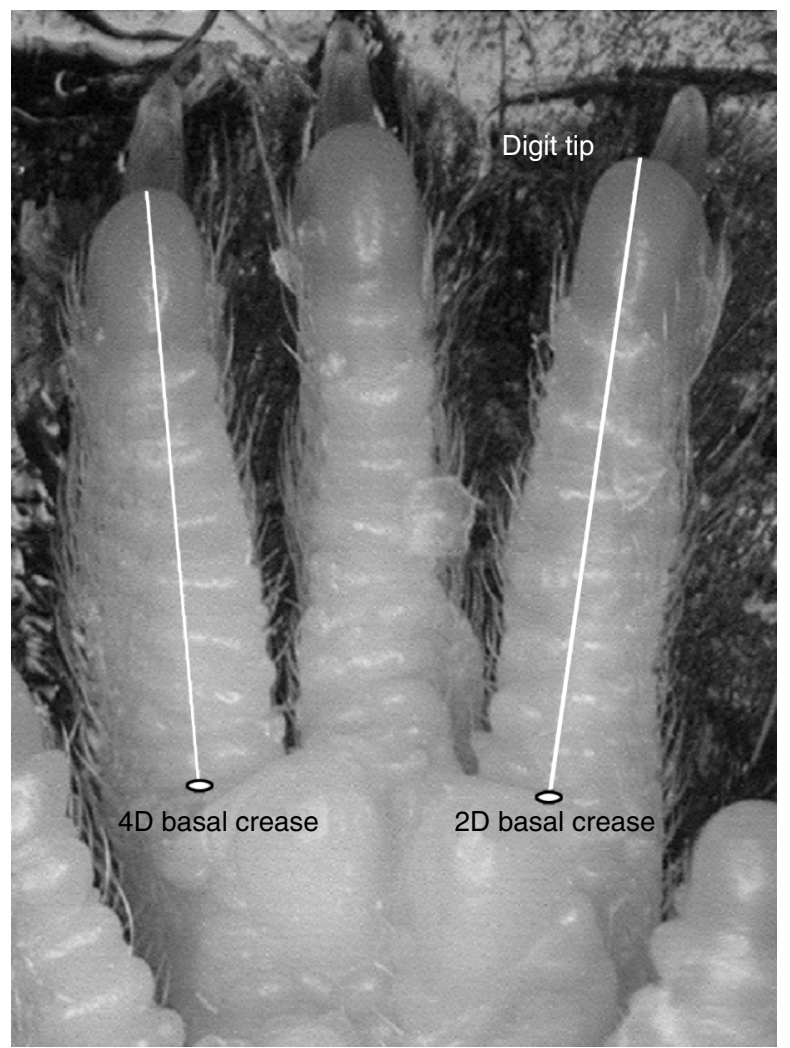

Figure 1: Photograph of the right rear paw palm from a female DBA/2J mouse illustrating the landmarks used to measure mice 2D:4D. advantage of this method of digit measurement, from the basal crease rather than from a pin pressed into the ' $V$ ' between digits as in Brown et al. (2002a), was that the depth of the webbing between digits appeared to vary considerably between strains, but the basal crease did not seem to vary in its location on the paw. Also, measuring in pixels on a digital image avoided having to estimate tenths of a millimeter on a plastic ruler, as was necessary in Brown et al. (2002a). Manning et al. (2003b) did not publish the methods used to obtain their data.

Images were divided equally and randomly between two scorers for measurement. We tested the inter-rater reliability of our technique on a set of 10 randomly selected hind paws. The inter-rater reliability between two scorers' measures for rear 2D:4D was highly significant $(r=0.92, \mathrm{df}=8$ and $P<0.001)$.

We used a two-way ANOva between subjects to analyze differences in digit ratio in the right rear paws and left rear paws and the difference between the right and left paws between strains and sexes. A Pearson's $r$ was used to examine the association between mean strain 2D:4D and sex differences of 2D:4D with the strain. Cohen's (1988) $d^{\prime}$ was used to estimate effect sizes for strain and sex effects. Cohen's $d^{\prime}$ represents the difference between two group means divided by the pooled standard deviation.

\section{Results}

Digit ratios differed significantly between the strains on the right $\left(F_{7,168}=6.01, P<0.001\right)$ and left $\left(F_{7,167}=4.80\right.$, $P<0.001$ ) rear paws (Fig. 2). We found no difference in digit ratio between the sexes across strains (right: $F_{1,168}=0.07, P=0.79$; left: $F_{1,167}=0.14, P=0.71$ ) or a strain-sex interaction (right: $F_{7,168}=0.57, P=0.78$; left: $F_{7,167}=0.89, P=0.52$ ). There was a non-significant trend of positive association between mean strain 2D:4D and the direction and magnitude of the sex differences in digit ratio within strains $\left(r_{7}=0.62, P=0.098\right)$. In other words, strains with higher digit ratios tended to have higher male digit ratios compared with female ratios, whereas strains with lower pooled digit ratios tended to have lower male digit ratios than female ratios. There was no relationship between digit ratio asymmetry (right ratio minus left ratio) and sex $\left(F_{1,167}=0.21, P=0.65\right)$, strain $\left(F_{7,167}=0.59, P=0.76\right)$ or a sex-strain interaction $\left(F_{7,167}=0.72, P=0.65\right)$.

The Cohen's (1988) effect sizes for sex differences were small $\left(d^{\prime}=0.023\right)$, even when strain effects were removed $\left(d^{\prime}=0.041\right)$. In comparison, the effect size of the difference between DBA/2 J and $\mathrm{C} 3 \mathrm{H} / \mathrm{HeJ}$ mice was large, $d^{\prime}=1.460$. A non-parametric bootstrap based on 1000 resamplings (Efron \& Tibshirani 1993) was used to calculate a $95 \% \mathrm{Cl}$ on the magnitude of the sex effect. The ratio of male to female scores for the right rear paws has a $95 \% \mathrm{Cl}$ of 0.987 to 1.015. When strain differences are partialled out, the $95 \%$ $\mathrm{Cl}$ on ratio of male to female scores was 0.989 to 1.015 and the $95 \% \mathrm{Cl}$ on the effect size for sex was $d^{\prime}=-0.25$ to 0.32 . 

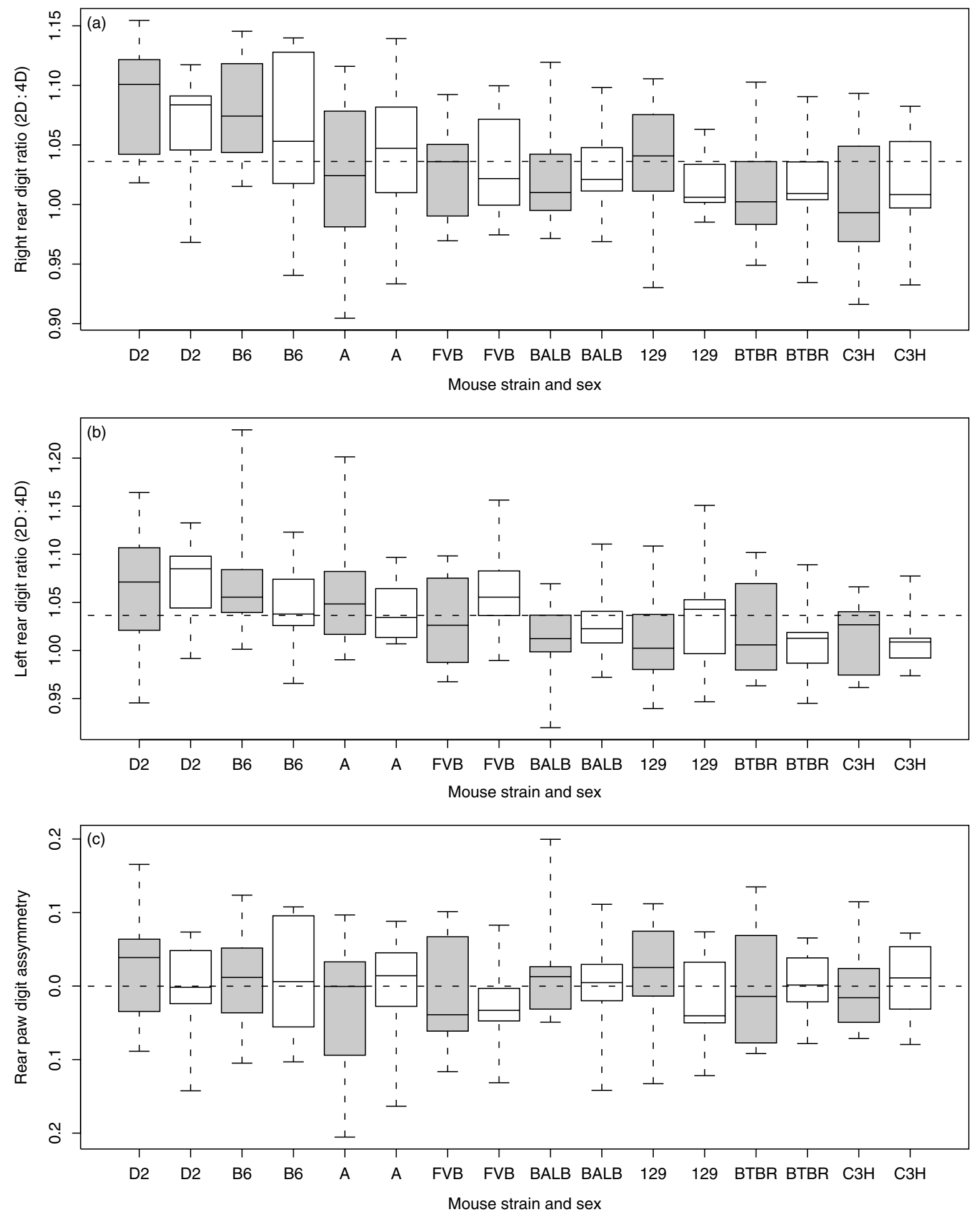

Figure 2: Digit ratio for eight inbred-mice strains by sex in right rear paws (a), left rear paws (b) and asymmetry between rear paws (c). Strains are DBA/2J (D2), C57BL/6J (B6), A/J (A), FVB/NJ (FVB), BALB/cByJ (BALB), 129S1/SvImJ (129), BTBR_+T_tf/tf (BTBR) and $\mathrm{C} 3 \mathrm{H} / \mathrm{HeJ}(\mathrm{C} 3 \mathrm{H})$. Gray boxes denote male mice and white boxes denote female mice for each strain. Boxes indicate the first and third quartiles whereas the median is marked with a horizontal line, whiskers extending to the most extreme datum. The dotted line represents the average digit ratio for all strains combined. 


\section{Discussion}

Digit ratios differed significantly between the inbred strains but not between the sexes. We also found no significant differences between sexes or strains in the lateral asymmetry in digit ratios. No such sex effect on digit ratio asymmetry exists in humans (Putz et al. 2004). The failure to find an overall sex effect is surprising. Brown et al. (2002a) found digit ratio to be sexually differentiated in the right rear paw only of outbred laboratory mice, whereas Manning et al. (2003b) found only the left rear paw to be sexually differentiated. We believe the sex effects found in these earlier studies to be real. Possible explanations for these different results include (1) different methods used to measure digit length, (2) different effective sample sizes and (3) different types of mice used.

Our scores cannot be compared directly to either previous study because of the different measuring methods used, but we do not believe that the difference in methods is the source of the difference in results. We estimated the effect size of sex from Brown et al. (2002a) to be $d^{\prime}=0.357$. This is larger than the $95 \% \mathrm{Cl}$ for the sex effect calculated from our data but not nearly as large as the effect sizes found between the most different strains in our study. This suggests that we had enough power to detect a sex effect had there been one. We found no clear trend in digit ratio between the sexes such that a larger sample size in our study would have produced a sex effect. We believe the reason we did not find a net sex effect is that this effect varies between strains. Strains with higher digit ratios tended to have sex effects in the opposite direction from strains with lower digit ratios. The small number of mice per strain may have provided insufficient power to detect a sex by strain interaction. Alternatively, inbreeding may somehow disrupt the sex differences in digit ratio.

Intrauterine position (IUP) (Clark \& Galef 1995; vom Saal \& Bronson 1980) may also contribute variability to digit ratios, masking the effect of sex. Pups which gestate between two males may have different digit ratios than those which gestate between two females. Interstrain variation in litter size may also contribute to IUP effects on mean strain digit ratio. There was no obvious correlation between mean litter size per strain or average sex ratio at weaning (data from mpd149.dat, the Mouse Phenome Database, q.v.) with either mean strain digit ratio or mean female digit ratio. However, only four strains used in our study also appear in the reproductive performance data set. Further research is warranted into the relationship between digit ratio, IUP and inter-strain variation.

We identified a number of traits in the Mouse Phenome Database (http://www.jax.org/phenome) to assess the potential of using inbred mice for investigating the association between digit ratio and behavioral traits thought to be influenced by developmental androgens. The mouse behavioral traits we chose seemed to correspond to human behavioral traits which have been shown to correlate with digit ratio in previous studies. Some of these assays may not model the human traits well, and caution is warranted in interpreting the results. Table 1 lists the behavioral mice traits, the corresponding human traits and the correlation

Table 1: The relationship between average inbred mouse strain digit ratios and behavioral traits compared with the existing digit ratiobehavioral trait relationships in humans

\begin{tabular}{|c|c|c|c|c|c|}
\hline \multirow[b]{3}{*}{ Trait measured } & \multicolumn{4}{|c|}{ Inbred mice } & \multirow{3}{*}{$\begin{array}{l}\text { Humans } \\
\text { Existing digit ratio-trait relationship }\end{array}$} \\
\hline & \multicolumn{3}{|c|}{ Digit ratio-trait correlation } & \multirow[b]{2}{*}{ Interpretation } & \\
\hline & $r$ & $d f$ & $P$ & & \\
\hline $\begin{array}{l}\text { Activity* } \\
\text { (total daily activity) }\end{array}$ & 0.52 & 12 & 0.058 & $\begin{array}{l}\text { Higher ratios: trend } \\
\text { toward increased activity }\end{array}$ & $\begin{array}{l}\text { Lower ratios: increased speed, } \\
\text { achievement and ability in sports } \\
\text { (Manning \& Taylor 2001) }\end{array}$ \\
\hline $\begin{array}{l}\text { Aggressiont } \\
\text { (average number of bites) }\end{array}$ & 0.65 & 14 & 0.006 & $\begin{array}{l}\text { Higher ratios: increased } \\
\text { aggression }\end{array}$ & $\begin{array}{l}\text { Lower ratios: increased } \\
\text { physical aggression } \\
\text { (Bailey \& Hurd 2004) }\end{array}$ \\
\hline $\begin{array}{l}\text { Anxiety } \ddagger \\
\text { (\% of time in open quadrants) }\end{array}$ & -0.45 & 12 & 0.11 & $\begin{array}{l}\text { Lower ratios: trend } \\
\text { toward more anxiety exhibited } \\
\text { toward more anxiety exhibited }\end{array}$ & $\begin{array}{l}\text { Higher ratios: more } \\
\text { trait depression exhibited } \\
\text { (A. A. Bailey and P. L. Hurd unpublished data) }\end{array}$ \\
\hline Systolic blood pressure§ & 0.52 & 12 & 0.055 & $\begin{array}{l}\text { Higher ratios: trend } \\
\text { toward increased }\end{array}$ & $\begin{array}{l}\text { Lower ratios: myocardial infarction } \\
\text { happens later in life } \\
\text { (Manning \& Bundred 2001) }\end{array}$ \\
\hline Body weight & -0.24 & 14 & 0.36 & No relationship & No relationship (Manning et al. 2000b) \\
\hline
\end{tabular}

Mice behavioral data for each strain were obtained from http://www.jax.org/phenome. The data sets (trait abbreviations in data sets) used were ${ }^{*}$ MPD:92 (tot_daily), 'MPD:160 (nbite), ${ }^{\ddagger}$ MPD:118 (pct_open), §MPD:104 (BP) and "MPD108 (bw). The correlations are between the average right rear digit ratios for males and females within each of the eight strains and the strain average for each behavioral trait. 
found between mean mice traits and the mean right rear digit ratios for each sex and strain combination in this study. As each of the mouse traits measured showed a strong trend, significant effect of sex or sex by strain interaction in the data sets used (sex effect: total daily activity $P<0.001$, blood pressure $P<0.051$ and body weight $P<0.001$; interaction: number of bites $P<0.06$ ), we analyzed each strain-sex mean as a separate datum. Although our eight strains produce fairly low statistical power, Table 1 summarizes a general tendency for those traits to correlate with digit ratio.

We chose to analyze the relationships between behavioral data and the right rear paw, because several authors have suggested that androgenization affects the right hand more than the left (Brown et al. 2002a; McFadden \& Shubel 2002; Williams et al. 2000). Digit ratio has consistently been shown to be more strongly differentiated on the right hand than on the left, in humans (Brown et al. 2002b; Lippa 2003; Manning et al. 1998; McFadden \& Shubel 2002; Williams et al. 2000), regardless of handedness (Williams et al. 2000), and in mice (Brown et al. 2002a) and in finches (Burley \& Foster 2004). Personality and behavioral traits also correlate more strongly with right hand digit ratio than left (Csatho et al. 2003a, 2003b; Williams et al. 2000; Williams et al. 2003).

It is difficult to interpret the results summarized in Table 1 without wondering whether smaller or larger ratios are the more masculine in mice. In humans, not only is there a sex difference in digit ratio but behavioral variation also correlates with digit ratio within sexes consistently with the sexual variation in that behavior. Mice strains did not show such a simple effect. Higher ratios in hind paws of mice were associated with more masculine behavioral traits such as increased aggression and increased activity. These effects are the opposite to that of previous studies with human hand digit ratios. Lower digit ratio in humans tends to correlate with more masculine traits such as increased physical aggression and increased speed, ability and achievement in sports (Bailey \& Hurd in press; Manning 2002a, 2002b; Manning \& Taylor 2001). In humans and two strains of mice, the average male digit ratio is lower than the average female digit ratio; however, foot digit ratio in humans and zebra finches shows reverse sexual differentiation (i.e. females have smaller ratios than males, Burley \& Foster 2004; McFadden \& Shubel 2002; Manning et al. 2003b).

\section{Conclusions}

The current findings show promise for investigating the relationship between developmental androgens, digit ratio and behavioral traits. Inbred-mice strains appear to provide large stable differences in baseline digit ratios. More research needs to be done both to elucidate the sex effect within strains and to correlate digit ratio with other morphological correlates of androgenization such as anogenital distance.

\section{References}

Bailey, A.A. \& Hurd, P.L. (2004) Finger length ratio (2D:4D) correlates with physical aggression in men but not in women. Biol Psychol doi: 10.1016/j.biopsycho.2004.05.001.

Brown, W.M., Finn, C.J. \& Breedlove, S.M. (2002a) Sexual dimorphism in digit-length ratios of laboratory mice. Anat Rec 267, 231-234.

Brown, W.M., Hines, M., Fane, B.A. \& Breedlove, S.M. (2002b) Maculinized finger length patterns in human males and females with congenital adrenal hyperplasia. Horm Behav $\mathbf{4 2}$, 380-386.

Burley, N.T. \& Foster, V.S. (2004) Digit ratio varies with sex, egg order, and strength of mate preference in zebra finches. Proc $R$ Soc Lond B Biol Sci 271, 239-244.

Chamberlain, N.L., Driver, E.D. \& Miesfeld, R.L. (1994) The length and location of CAG trinucleotide repeats in the androgen receptor $n$-terminal domain affect transactional function. Nucleic Acids Res 15, 3181-3186.

Clark, M.M. \& Galef, B.G. (1995) Prenatal influences on reproductive life history strategies. TREE 10, 151-153.

Cohen, J. (1988) Statistical power analysis for the behavioral sciences. Lawrence Earlbaum Associates, NJ.

Csatho, A., Osvath, A., Bicsak, E., Karadi, K., Manning, J. \& Kallai, J. (2003a) Sex role identity related to the ratio of second to fourth digit length in women. Biol Psychol 62, 147-156.

Csatho, A., Osvath, A., Karadi, K., Bicsak, E., Manning, J. \& Kallai, J. (2003b) Spatial navigation related to the ratio of second to fourth digit length in women. Learning and individual differences 13, 239-249.

Efron, B. \& Tibshirani, R.J. (1993) An introduction to the bootstrap. Chapman \& Hall, NY.

Flint, J. (2003) Analysis of quantitative trait loci that influence animal behavior. J Neurobio/ 54, 46-77.

Garn, S.M., Burdu, A.R., Babler, W.J. \& Stinson, S. (1975) Early prenatal attainment of adult metacarpal-phalangeal rankings and proportions. Am J Phys Anthropol 43, 327-332.

Kazemi-Esfarjani, P., Trifiro, M.A. \& Pinski, L. (1995) Evidence for a repressive function of the long polyglutamine tract in the human androgen receptor: possible pathogenic relevance for the (CAG) n-expanded neuropathies. Hum Mol Genet 4, 523-527.

Kondo, T., Zakany, J., Innis, W.J. \& Duboule, D. (1997) Of fingers, toes, and penises. Nature 390, 29.

Lippa, R.A. (2003) Are 2D:4D finger-length ratios related to sexual orientation? Yes for men, no for women. J Pers Soc Psych 85 (1), 179-188.

Manning, J.T. (2002a) Digit ratio: a pointer to fertility, behaviour, and health. Rutgers U Press, New Brunswick, NJ.

Manning, J.T. (2002b) The ratio of 2nd to 4th digit length and performance in skiing. J Sports Med Phys Fitness 42, 446-450.

Manning, J.T. \& Bundred, P.E. (2001) The ratio of second to fourth digit length and age at first myocardial infarction in men: a link with testosterone. Br J Cardio/ 8, 720-723.

Manning, J.T. \& Taylor, R.P. (2001) Second to fourth digit ratio and male ability in sport: implications for sexual selection in humans. Evol Hum Behav 22, 61-69.

Manning, J.T., Scott, D., Wilson, J. \& Lewis-Jones, D.I. (1998) The ratio of 2 nd to 4th digit length: a predictor of sperm numbers and concentration of testosterone, leutenizing hormone and oestrogen. Hum Reprod 1311, 3000-3004.

Manning, J.T., Barley, L., Walton, J., Lewis-Jones, D., Trivers, R.L., Singh, D. et al. (2000a) The 2nd:4th digit ratio, sexual dimorph- 
ism, population differences, and reproductive success: evidence for sexually antagonistic genes. Evol Hum Behav 21, 163-183.

Manning, J.T., Trivers, R.L., Thornhill, R. \& Singh, D. (2000b) The 2nd:4th digit ratio and asymmetry of hand performance in Jamaican children. Laterality 5, 121-132.

Manning, J.T., Bundred, P.E., Newton, D.J. \& Flanigan, B.F. (2003a) The second to fourth digit ratio and variation in the androgen receptor gene. Evol Hum Behav 24, 399-405.

Manning, J.T., Callow, M. \& Bundred, P.E. (2003b) Finger and tow ratios in humans and mice: Implications for the aetiology of diseases influenced by HOX genes. Med Hypotheses 60 , 340-343.

McFadden, D. \& Shubel, E. (2002) Relative lengths of fingers and toes in human males and females. Horm Behav 42, 492-500.

Okten, A., Kalyoncu, M. \& Yaris, N. (2002) The ratio of secondand fourth-digit lengths and congenital adrenal hyperplasia due to 21-hydroxylase deficiency. Early Hum Dev 70, 47-54.

Peichel, C.L., Prabhakaran, B. \& Vogt, T.F. (1997) The mouse ulnaless mutation deregulates posterior hoxd gene expression and alters appendicular patterning. Development 124, 3481-3492.

Peters, M., Tan, U., Kang, Y., Teixeira, L. \& Mandal, M. (2002) Sexspecific finger-length patterns linked to behavioral variables: consistency across various human populations. Percept Mot Skills 94, 171-181.

Putz, D.A., Gaulin, S.J.C., Sporter, R.J. \& McBurney, D.H. (2004) Sex homones and finger length: What does 2D:4D indicate? Evol Hum Behav 25, 182-199. vom Saal, F.S. \& Bronson, F.H. (1980) Sexual characteristics of adult female mice are correlated with their blood testosterone levels during prenatal development. Science 208, 597-599.

Wahlsten, D., Metten, P., Phillips, T.J., Boehm, S.L., BurkhartKasch, S., Dorow, J., Doerksen, S., Downing, C., Fogarty, J., Rodd-Henricks, K., Hen, R., McKinnon, C.S., Merrill, C.M., Nolte, C., Schalomon, M., Schlumbohm, J.P., Sibert, J.R., Wenger, C.D., Dudek, B.C. \& Crabbe, J.C. (2003) Different data from different labs: lessons from studies of geneenvironment interaction. J Neurobio/ 54, 283-311.

Williams, T.J., Pepitone, M.E., Christensen, S.E., Cooke, B.M., Huberman, A.D., Breedlove, N.J., Breedlove, T.J., Jordan, C.L. \& Breedlove, S.M. (2000) Finger length ratios and sexual orientation. Nature 404, 455-456.

Williams, J.H.G., Greenhalgh, K.D. \& Manning, J.T. (2003) Second to fourth finger ratio and possible precursors of developmental psychopathology in preschool children. Early Hum Dev $\mathbf{7 2}$ 57-65.

\section{Acknowledgments}

Research funding by NIH Grant (R01 AA12714) to DW and NSERC (Canada) Discovery Grant (249685 and 45825) to PLH and DW, respectively. We thank Patricia Gongal, Sarrah Mohamedbhai, Alisha Brown, Brandy Moisan and Sean Cooper for assistance in collecting the data. 